\title{
AnnotationBustR: An R package to extract subsequences from GenBank annotations
}

\author{
Samuel R. Borstein ${ }^{\text {Corresp., }}{ }^{1}$, Brian C. O'Meara ${ }^{1}$ \\ ${ }^{1}$ Department of Ecology \& Evolutionary Biology, University of Tennessee - Knoxville, Knoxville, Tennessee, United States \\ Corresponding Author: Samuel R. Borstein \\ Email address: sborstei@vols.utk.edu
}

Background. DNA sequences are pivotal for a wide array of research in biology. Large sequence databases, like GenBank, provide an amazing resource to utilize DNA sequences for large scale analyses. However, many sequence records on GenBank contain more than one gene or are portions of genomes. Inconsistencies in the way genes are annotated and the numerous synonyms a single gene may be listed under provide major challenges for extracting large numbers of subsequences for comparative analysis across taxa. At present, there is no easy way to extract portions from many GenBank accessions based on annotations where gene names may vary extensively. Results. The R package AnnotationBustR allows users to extract sequences based on GenBank annotations through the ACNUC retrieval system given search terms of gene synonyms and accession numbers. AnnotationBustR extracts subsequences of interest and then writes them to a FASTA file for users to employ in their research endeavors. Conclusion. FASTA files of extracted subsequences and accession tables generated by AnnotationBustR allow users to quickly find and extract subsequences from GenBank accessions. These sequences can then be incorporated in various analyses, like the construction of phylogenies to test a wide range of ecological and evolutionary hypotheses. 
1 AnnotationBustR: An R package to extract subsequences from GenBank annotations

2 Samuel R. Borstein ${ }^{1}$ and Brian C. O’Meara ${ }^{1}$

$3 \quad{ }^{1}$ Department of Ecology \& Evolutionary Biology, University of Tennessee, Knoxville, TN, USA

4

5 Corresponding Author:

6 Samuel R. Borstein 1

7569 Dabney, University of Tennessee, Knoxville, TN 37996-1610, USA

8 Email address: sborstei@vols.utk.edu

9 


\section{Abstract}

Background. DNA sequences are pivotal for a wide array of research in biology. Large sequence databases, like GenBank, provide an amazing resource to utilize DNA sequences for large scale analyses. However, many sequence records on GenBank contain more than one gene or are portions of genomes. Inconsistencies in the way genes are annotated and the numerous synonyms a single gene may be listed under provide major challenges for extracting large numbers of subsequences for comparative analysis across taxa. At present, there is no easy way to extract portions from many GenBank accessions based on annotations where gene names may vary extensively.

Results. The R package AnnotationBustR allows users to extract sequences based on GenBank annotations through the ACNUC retrieval system given search terms of gene synonyms and accession numbers. AnnotationBustR extracts subsequences of interest and then writes them to a FASTA file for users to employ in their research endeavors.

Conclusion. FASTA files of extracted subsequences and accession tables generated by AnnotationBustR allow users to quickly find and extract subsequences from GenBank accessions. These sequences can then be incorporated in various analyses, like the construction of phylogenies to test a wide range of ecological and evolutionary hypotheses.

\section{Introduction}

The use of DNA sequence data is vital for a wide variety of research in evolutionary biology and ecology. Molecular phylogenies, which rely on DNA sequences for their construction, are extremely prevalent in biological research. Whether being used to correct for shared ancestry among organisms (Felsenstein, 1985), or to test hypotheses related phylogeography (Avise et al., 1987), diversification (Hey, 1992; Maddison, 2006), and trait evolution (Hansen, 1997; Bollback, 2006), phylogenies are required. Additionally, the use of phylogenies is important in community ecology to place systems into an evolutionary framework (Webb et al., 2002; Cavender-Bares et al., 2009). The construction of molecular phylogenies for systematic purposes is also a popular tool for taxonomists to identify new taxa and classify organisms (De Queiroz \& Gauthier, 1994; Tautz et al., 2003). Some DNA sequences, like the mitochondrial gene cytochrome oxidase subunit I (COI), have utility as a method to identify and catalog species using DNA barcoding (Hebert et al., 2003; Ratnasingham \& Hebert, 2007; Ratnasingham \& Hebert, 2013).

Sequence databases like GenBank provide a valuable resource for using DNA sequence data to test evolutionary and ecological hypotheses. With the reduction in cost of DNA sequencing and the advancement of methods to analyze sequence data, the amount of sequence data available for use is growing at a rapid pace. Given that GenBank has over one-trillion sequences from over 370,000 species (Benson et al., 2017) and recent advances in methods to create massive phylogenies using either super-matrix (Driskell et al., 2004; Ciccarelli et al., 2006) or mega-phylogeny approaches (Smith et al., 2009; Izquierdo-Carrasco et al., 2014), the ability to generate large DNA sequence data sets for comparative analyses has become fairly common (Leslie et al., 2012; Rabosky et al., 2013; Spriggs et al., 2014; Zanne et al., 2014; Shi \& Rabosky, 2015). Additionally, sequence retrieval with command line utilities like National Center for Biotechnology Information's eutils (NCBI Resource Coordinators, 2017) as well as within common scripting environments for biological analyses, like $\mathrm{R}$ (R Development Core Team, 2018), Perl (Perl Development Team, 2017), and Python (Python Software Foundation, 
54

55

56

57

58

59

60

61

62

63

64

65

66

67

68

69

70

71

72

73

74

75

76

77

78

79

80

81

82

83

84

85

86

87

88

89

90

91

92

93

94

95

96

2016), are made possible with packages like ape (Paradis et al., 2004), rentrez (Winter, 2016), reutils (Schofl, 2015), seqinr(Charif \& Lobry, 2007), Bioperl (Stajich et al., 2002), and Biopython (Chapman \& Chang, 2000).

While GenBank provides a wealth of sequence data for researchers to use, some of it is rather difficult to manipulate into a useful form. For example, some sequences may be concatenated together, or the only gene sequence available for a species for the locus of interest may be within a mitochondrial or chloroplast genome. At the time of writing, GenBank has 70,048 complete or partial mitochondrial genomes, 4,698 chloroplast genomes, and a simple search for sequences containing concatenated coding sequences and tRNAs resulted in 286,538 additional sequences exclusive of the previously mentioned sequences. Although GenBank's annotation system provides a means to see where a locus of interest is in a genome or concatenated sequence and provides the ability to download it manually, this is extremely time consuming when many accessions are involved and not a feasible way to extract mass amounts of sequence data for use in research. While alternative sequence databases exist, especially for popular loci utilized for DNA barcoding and microbial community identification (ex. COI, 16S, etc.), these databases typically are extremely focused on just a few loci (DeSantis et al., 2006; Ratnasingham \& Hebert, 2007; Cole et al., 2013). While these databases typically house data from GenBank, they may not have complete overlap with sequence data on GenBank, such as those from complete organelle sequences (ex. GenBank KR150862.1 for the mitochondrial genome of Bujurquina mariae, which is a species not included in the Barcode of Life Database (BOLD) even though the GenBank accession has a record for cytochrome oxidase subunit I ).

Another major challenge to obtaining large amounts of sequence data is the highly variable nomenclature of gene names. Most genes have several alternative names and symbols that are present in sequence databases. Among distant taxa, it is common for homologous genes to vary considerably in nomenclature (Tuason et al., 2003). Even within a group of closely related taxa or within a single taxon itself, how genes are annotated may differ substantially from record to record and a wide variety of alternative gene names may be found for a single gene (Morgan et al., 2004; Fundel \& Zimmer, 2006). This poses serious problems when searching through databases for molecular sequence data (Mitchell et al., 2003; Tamames \& Valencia, 2006).

Here we present the R package AnnotationBustR to address the issues discussed above. AnnotationBustR reads GenBank annotations in $\mathrm{R}$ and pulls out the gene(s) of interest given a set of search terms and a vector of taxon accession numbers supplied by the user. It then writes the sequence for the gene(s) of interest to FASTA formatted files for each locus that users can then use in further analyses. For a more in-depth introduction to using AnnotationBustR users should consult the vignette in R through vignette ("AnnotationBustR-vignette"), which provides instructions on how to use the different functions and their respective options. Other details about the package can be accessed through the documentation via help ("AnnotationBustR").

\section{Description}

AnnotationBustR is written in R (R Development Core Team, 2017), a popular language for analyzing biological data, and requires $\mathrm{R}$ version 3.4 or higher. It uses the existing $\mathrm{R}$ packages ape (Paradis et al., 2004) and seqinr (Charif \& Lobry, 2007). AnnotationBustR uses 
97 seqinr's interface to the online ACNUC database to extract gene regions of interest from 98 concatenated gene sequences or genomes (Gouy et al., 1985; Gouy \& Delmotte, 2008). ACNUC 99 is a database and retrieval system for molecular sequence data maintained by the Pôle Bio-

Informatique Lyonnais, which provides access to GenBank data and allows for easy access and manipulation of complex sequences, such as trans-spliced genes that may be on opposite strands of DNA. A list of the currently implemented commands is given in Table 1 and a flow chart of function usage is shown in Figure 1.

Table 1: Functions and data included in the package AnnotationBustR.

\begin{tabular}{|l|l|}
\hline Function/Data Name & Description \\
\hline AnnotationBust & $\begin{array}{l}\text { Writes found subsequences for loci of interest to a FASTA } \\
\text { file for a vector of GenBank accessions and writes a } \\
\text { corresponding accession table. }\end{array}$ \\
\hline data (cpDNAterms) & Loads a data frame of search terms for chloroplast genes. \\
\hline data (mtDNAterms) & $\begin{array}{l}\text { Loads a data frame of search terms for animal } \\
\text { mitochondrial genes. }\end{array}$ \\
\hline data (mtDNAtermsPlants) & $\begin{array}{l}\text { Loads a data frame of search terms for plant mitochondrial } \\
\text { genes. }\end{array}$ \\
\hline data (rDNAterms) & $\begin{array}{l}\text { Loads a data frame of search terms for ribosomal DNA } \\
\text { genes and spacers. }\end{array}$ \\
\hline FindLongestSeq & $\begin{array}{l}\text { Finds the longest sequence for each species in a set of } \\
\text { GenBank accession numbers. }\end{array}$ \\
\hline MergeSearchTerms & $\begin{array}{l}\text { Merges two or more data frames containing search terms of } \\
\text { features to extract into a single data frame. }\end{array}$ \\
\hline
\end{tabular}

The main function of AnnotationBustR, AnnotationBust, takes a vector of accession numbers and a data frame of synonym search terms to extract loci of interest and writes them to a FASTA formatted file. This function also returns an accession table of all the loci of interest and the corresponding accession numbers the loci were extracted from for each species that can then be written to a csv file. Users can specify that duplicate genes should be extracted as well. If extracting coding sequences, users can also specify if they would like to translate the sequence into the corresponding peptides by specifying the appropriate GenBank numerical translation code. 
113

114

115

116

117

118

119

120

121

122

123

124

125

126

127

128

129

130

131

132

133

134

135

136

137

138

139

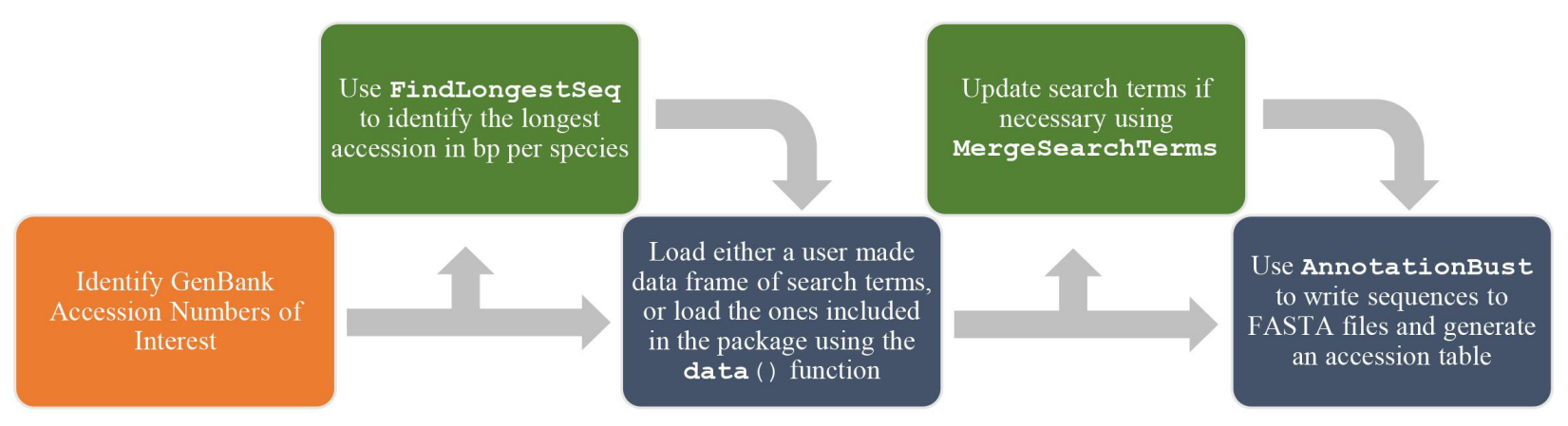

Figure 1: Flow chart of functions for a complete usage of AnnotationBustR. Blue boxes indicate a step using the package AnnotationBustR while orange boxes represent steps that need to be completed outside of AnnotationBustR. Boxes in green represent optional steps in the AnnotationBustR pipeline.

We have included pre-made data frames with search terms in AnnotationBustR for animal and plant mitochondrial genomes, chloroplast genomes, and rDNA. These can be used to easily extract DNA barcodes, like cytochrome oxidase subunit I (COI) for animals in mitochondrial genomes (Hebert et al., 2003), the internal transcribed spacers (ITS) in rDNA for fungi and plants (Kress et al., 2005; Schoch et al., 2012), and maturase K (matK) and ribulosebisphosphate carboxylase (rbcL) genes in the chloroplast genome of plants (Hollingsworth et al., 2009). These pre-made data frames consist of three columns with the column Locus containing the output file name, Type containing the type of sequence it is (i.e. CDS, tRNA, rRNA, misc_RNA, D-loop, etc.), and the third column, Name, containing a possible synonym of the loci to search for. For example, for cytochrome oxidase subunit I, GenBank includes gene names of COI, CO1, COX1, cox1, COXI, cytochrome c oxidase subunit I, and COX-I. An additional column, IntronExonNumber, is used to specify the intron or exon number for extracting introns and exons. These search terms can be loaded into the workspace using the data() function. Annotation files for each accession are read in through seqinr and regular expressions matching of the synonyms provided by the user to the feature annotations are performed to identify the subsequence to extract. As certain loci may have numerous synonymous listings in GenBank feature tables that may not be included in the pre-made data frames of search terms, AnnotationBustR has the function MergeSearchTerms which allows users to add additional search terms to a pre-existing data frame of search terms if users follow the basic column formatting stated above. An additional feature of AnnotationBustR is the function FindLongestSeq which finds the longest sequence for each species in a set of GenBank accessions. 


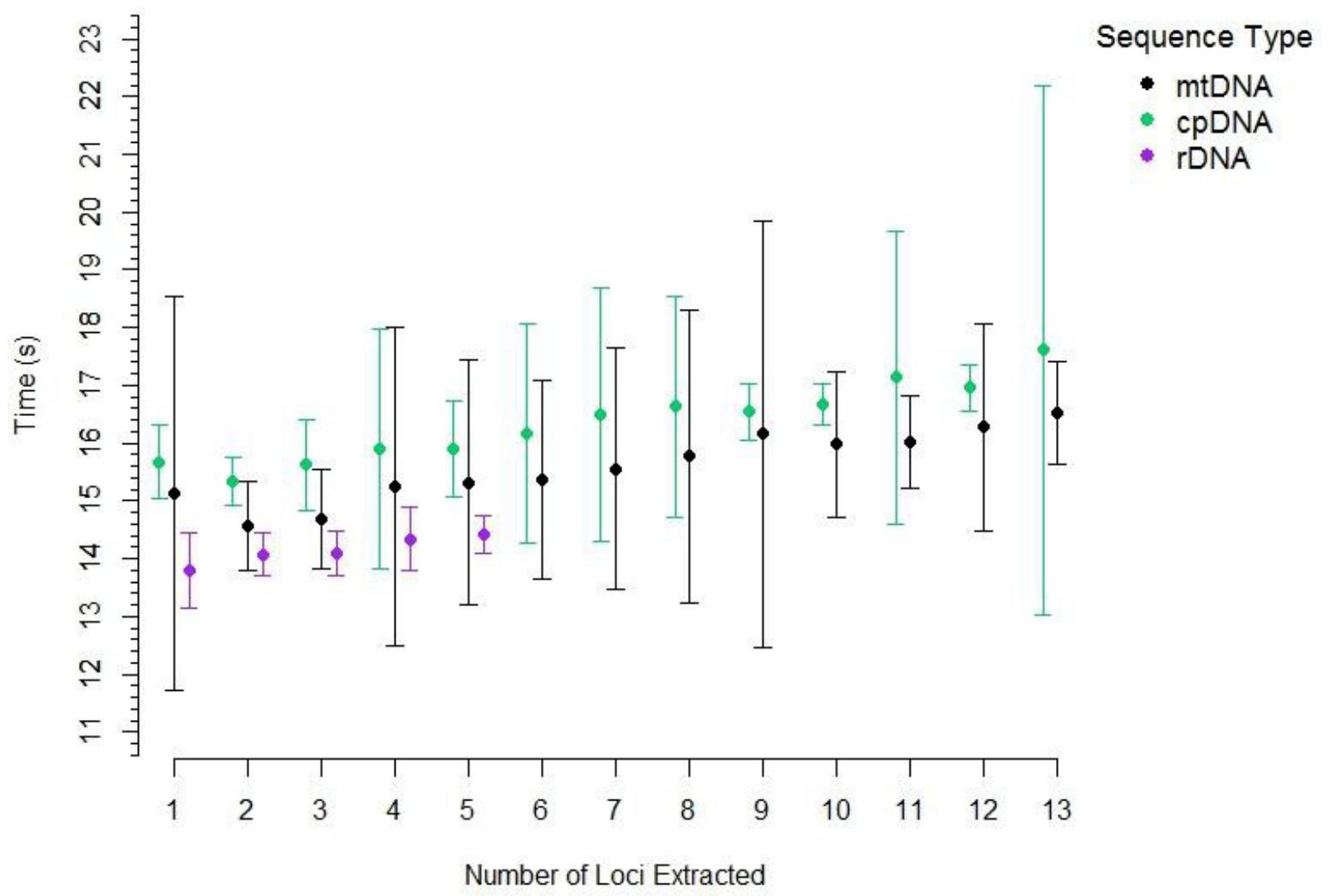

140

141

142

143

144

145

146

147

148

149

150

151

152

153

154

155

156

157

158

159

160

161

Figure 2: Timings of subsequence extraction using AnnotationBust for thirteen metazoan mitochondrial coding sequences (black), thirteen chloroplast coding sequences (green), and five metazoan rDNA subsequence (purple). Points represent the mean time in seconds with bars representing $+/$ - one standard deviation.

To demonstrate the performance of AnnotationBustR, we timed how long it took to extract thirteen popular coding sequences from 100 chloroplast genomes, the thirteen coding sequences from 100 metazoan mitochondrial genomes, and the three ribosomal RNA genes and internal transcribed spacers 1 and 2 from 100 metazoan rDNA sequences (Figure 2, see code in Supplemental Data S1). Timings were performed for each accession number starting with the extraction of a single locus and progressively adding an additional locus until all targeted loci were extracted for that accession. Timing trials were performed on a Windows desktop with a $3.8 \mathrm{GHz}$ Intel Core 17 processor and $64 \mathrm{~GB}$ of RAM. For each accession, we timed how long it took to extract one through the full number of subsequences sought. Our timings indicate that AnnotationBustR can efficiently extract these loci into FASTA files and that performance scales well as the number of loci to extract increases. Our timings also scale well in terms of sequence input size, as there is over variation in mean input sequence size between the rDNA (1,140 bp), mtDNA (16,560 bp), and cpDNA (149,971 bp) sequences. From profiling the code, it appears that the variation in the mean times to extract sequences is related to speed of the ACNUC server in returning responses to requests. While we did perform timing trials on a machine with a decent amount of RAM, AnnotationBustR is not very taxing on memory. The peak RAM usage of the AnnotationBust function during extraction for the largest dataset, the chloroplast 
162

163

164

165

166

167

168

169

170

171

172

173

174

175

176

177

178

179

180

181

182

183

184

185

186

187

188

189

190

191

192

193

194

195

196

197

198

199

200

201

202

203

204

205

genomes (average size of $149,971 \mathrm{bp}$ ), was only 87.35 megabytes when profiled using the $\mathrm{R}$ package peakRAM (Quinn, 2017).

AnnotationBustR is available through CRAN (https://cran.rproject.org/package $=$ AnnotationBustR) and is developed on GitHub (https://github.com/sborstein/AnnotationBustR). New extensions in development and fixes can be seen under the issues section on the packages GitHub page. Active lists of synonyms to be implemented in the next version are available on our github site in the issues section, as we gather more from users. While ACNUC currently only provides access to RefSeq virus sequences from the RefSeq database, we plan to add compatibility in the future for RefSeq accessions if access to other RefSeq databases is made available in seqinr.

\section{Examples}

We provide an example of the utility of sequences obtained using AnnotationBustR relative to single sequences on GenBank in North American leuciscine minnows. This group was chosen as they have been widely sequenced, both for whole mitochondrial genomes as well as single mitochondrial genes. Specifically, we extracted 12S rRNA, 16S rRNA, COI, CYTB, and ND2 genes from sixty species of leuciscine minnows and one outgroup, the zebrafish (Danio rerio). These five genes were chosen as individual gene sequences have also been regularly sequenced in this clade for phylogenetic studies (Bufalino \& Mayden, 2010; April et al., 2011; Schoenhuth et al., 2012). We used PHLAWD v. 3.3 (Smith et al., 2009) to identify GenBank accessions to download for single genes that had the best coverage and identity for each species. PHLAWD uses BLAST (Altschul et al., 1990) restricted to a specified clade of interest to identify the best sequence in terms of coverage and identity for each species relative to a set of known bait sequences. We specified sequences had to have at minimum of at least 20 percent identity and coverage to be kept in the dataset of sequences accessed from GenBank on April $16^{\text {th }}, 2018$. Multiple sequence alignment of sequences obtained from mitochondrial genomes using AnnotationBust $R$ and of individual GenBank genes was performed using MAFFT v. 7.402 (Katoh \& Standley, 2013). Phylogenetic reconstruction and bootstrap analysis was performed in RAxML v. 8.2 under the GTRGAMMA model of sequence evolution with the alignment partitioned by gene (Stamatakis, 2014)(supplemental data S1).

The alignment constructed using sequences extracted from mitochondrial genomes using AnnotationBust $R$ was $6,537 \mathrm{bp}$ and slightly longer than the 6,508 bp alignment constructed from individual GenBank sequences. The total number of sequences was 305 (extracted from the sixty-one mitogenomes) and 198, in the AnnotationBustR and GenBank alignments respectively. The most notable difference between the two alignments is the amount of missing data. The GenBank alignment was only $37.72 \%$ complete relative to the $97.59 \%$ complete AnnotationBustR alignment. The disparity in number of sequences and completeness of alignments is due to some species only having sequence data for a locus within a mitochondrial genome or other concatenated sequence and not as a single sequence on GenBank. While we do recover slightly different topologies between the datasets, we do find that the phylogeny reconstructed from the AnnotationBustR extracted sequences has higher bootstrap support among shared bipartitions relative to the tree constructed from individual GenBank sequences, with shared bipartitions amongst the phylogenies having an average of $93.69 \%$ and $81.56 \%$ bootstrap support in the AnnotationBustR and GenBank trees respectively (Figure 3). Both tree topologies are similar to those recovered in other phylogenetic studies of leuciscine minnows (Bufalino \& 
206

207

208

209

210

211

212

Mayden, 2010; Hollingsworth et al., 2013; Martin \& Bonett, 2015). Our results may not necessarily reflect any aspect of quality of sequence data used, but rather quantity. For example, all the sequences extracted using AnnotationBustR are the complete sequence for that gene. This is not necessarily true of the single gene sequences obtained from GenBank, where some species only have a partial sequence for a gene. Additionally, some species only have sequence data for certain genes as a mitochondrial genome accession and not necessarily have any other accession for that gene.

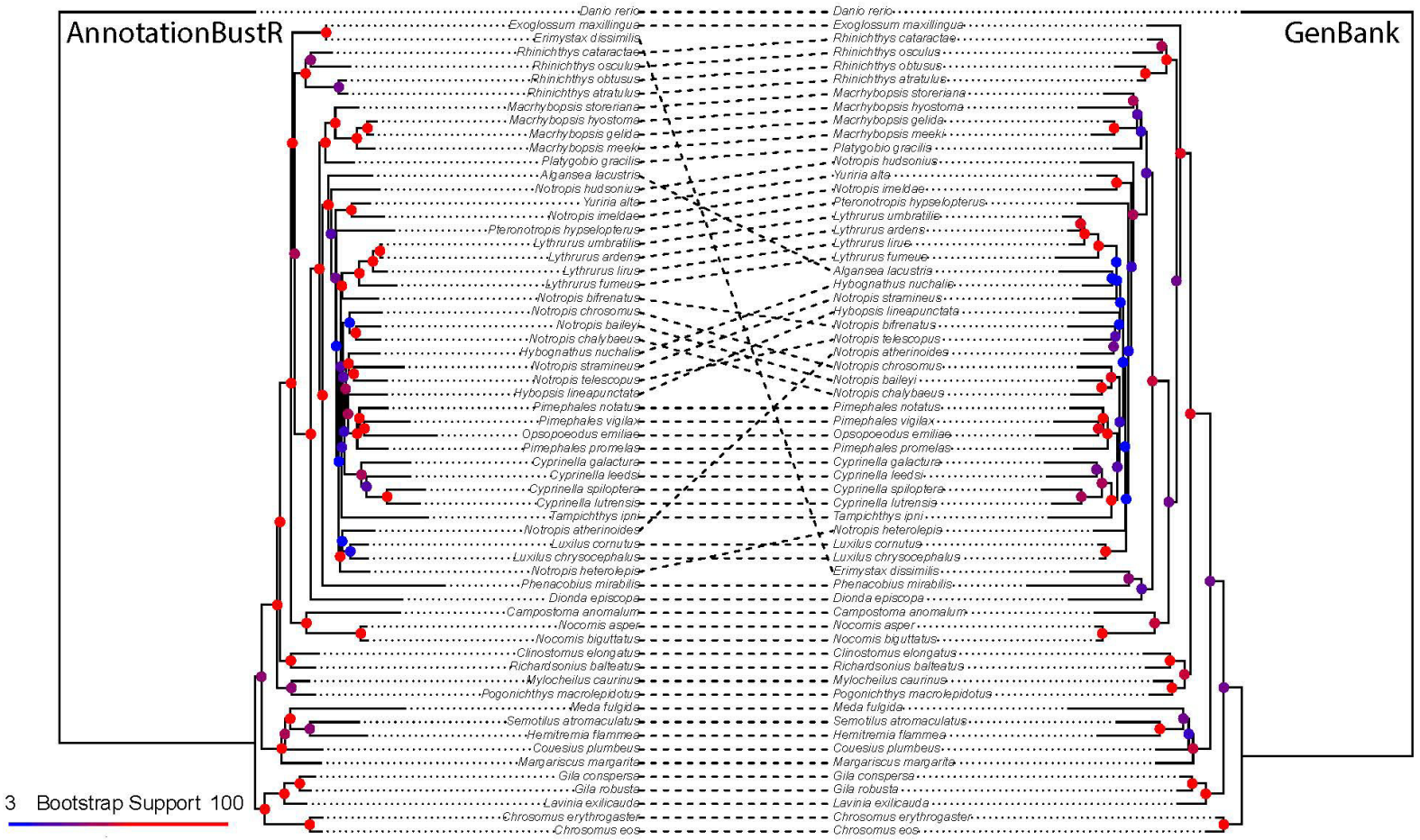

213

214

215

216

217

218

219

220

221

222

223

224

225

226

227

228

229

230

Figure 3: Phylogeny of 60 North American leuciscine minnows from sequences extracted using AnnotationBustR (left) and from individual GenBank sequences (right). Circles at nodes represent bootstrap support, with warmer colors representing higher bootstrap support and cooler colors representing lower bootstrap support.

\section{Conclusions}

AnnotationBustR provides a way for users to extract subsequences from concatenated sequences, plastid, and mitochondrial genomes where gene names for subsequences may vary substantially. The major limitation to the functionality of AnnotationBustR is that it is only as good as the annotations in the features table it is using for extraction. For instance, some concatenated sequences do not have the individual gene positions annotated for the record and just state that it contains the genes, therefore making it impossible to extract a gene from it (ex. GenBank KM260685.1. GenBank KT216295.1). Additionally, some loci may be present in the sequence yet missing from the features table completely (ex. mitochondrial D-loop missing in GenBank KU308536.1). Another limitation is that some popular loci such as intergenic spacers and some introns are not always annotated in the features table, making them impossible to extract. A good example of this is the trnH-psbA intergenic spacer, a proposed locus for plant DNA barcodes (Kress et al., 2005). As AnnotationBustR will extract all sequences within the 
231

232

233

234

235

236

237

238

239

240

241

242

243

244

245

246

247

248

249

250

251

252

253

254

255

256

257

258

259

260

261

262

263

264

265

266

267

268

269

270

271 annotations it finds that fit the supplied search terms, it is not a guarantee that these sequences are properly annotated, as annotation errors in sequence data are a known problem (Ben-Shitrit et al., 2012). Users will have to use due diligence on the sequences to check for incorrect annotations, mislabeled taxa, paralogs, chimeric sequences, and so forth. Alternative methods for extracting concatenated sequences could involve aligning them to known bait sequences of interest using a program like BLAST (Altschul et al., 1990). This can be automated by writing scripts to use BLAST locally or through NCBI. This represents a different approach from ours, relying on sequence similarity rather than annotations (PHLAWD builds on this but adds additional checks). While this can be useful, it potentially could be problematic as sequences could potentially align to non-orthologous sequences, which may cause issues with downstream analyses (Lassmann \& Sonnhammer, 2005). At large phylogenetic scales, bait sequences may blast to paralogous copies rather than to orthologous sequences of more distantly related taxa in the clade. Nonetheless, BLAST and tools building on it represent historically useful approaches for creating a matrix of sequences that may be quite complementary to use of AnnotationBustR as another quality control check in the curation of large molecular sequence datasets.

While eutils and packages in popular programming language can provide access to sequence data, and in some cases access to subsequences, they require complex query language. The R package we have developed provides a simple way for users to extract subsequences that have variable annotations by supplying a vector of accessions and search terms, either the ones included in the package or their own curated set. While R may not be as widely used as Python or Perl as a bioinformatics platform, it is a widely popular scripting language and we feel that this package fills a need in the R community.

\section{Citation}

Researchers publishing a paper that has used AnnotationBustR should cite this article and indicate the version of the package they are using. Package citation information can be obtained using citation("AnnotationBustR").

\section{Acknowledgements}

We thank members of the O'Meara lab, Cedric Landerer, and Christopher Peterson for helpful discussions while developing the package and Orlando Schwery and Frankie West for beta testing. We thank the seqinr package authors for technical help related to their package. We thank three anonymous reviewers for their helpful feedback on improvements for the paper and the software.

\section{References}

Altschul SF, Gish W, Miller W, Myers EW, and Lipman DJ. 1990. Basic local alignment search tool. Journal of Molecular Biology 215:403-410. 10.1006/jmbi.1990.9999

April J, Mayden RL, Hanner RH, and Bernatchez L. 2011. Genetic calibration of species diversity among North America's freshwater fishes. Proc Natl Acad Sci U S A 108:10602-10607. 10.1073/pnas.1016437108

Avise JC, Arnold J, Ball RM, Bermingham E, Lamb T, Neigel JE, Reeb CA, and Saunders NC. 1987. Intraspecific phylogeography: the mitochondrial DNA bridge between population genetics and systematics. Annual Review of Ecology and Systematics:489-522. 
Ben-Shitrit T, Yosef N, Shemesh K, Sharan R, Ruppin E, and Kupiec M. 2012. Systematic identification of gene annotation errors in the widely used yeast mutation collections. Nature methods 9:373378.

Benson DA, Cavanaugh M, Clark K, Karsch-Mizrachi I, Lipman DJ, Ostell J, and Sayers EW. 2017. GenBank. Nucleic Acids Res 45:D37-D42. 10.1093/nar/gkw1070

Bollback JP. 2006. SIMMAP: stochastic character mapping of discrete traits on phylogenies. Bmc Bioinformatics 7:88. 10.1186/1471-2105-7-88

Bufalino AP, and Mayden RL. 2010. Phylogenetic evaluation of North American Leuciscidae (Actinopterygii: Cypriniformes: Cyprinoidea) as inferred from analyses of mitochondrial and nuclear DNA sequences. Systematics and Biodiversity 8:493-505. 10.1080/14772000.2010.516029

Cavender-Bares J, Kozak KH, Fine PV, and Kembel SW. 2009. The merging of community ecology and phylogenetic biology. Ecology Letters 12:693-715.

Chapman B, and Chang J. 2000. Biopython: Python tools for computational biology. ACM Sigbio Newsletter 20:15-19.

Charif D, and Lobry JR. 2007. SeqinR 1.0-2: a contributed package to the R project for statistical computing devoted to biological sequences retrieval and analysis. Structural approaches to sequence evolution: Springer, 207-232.

Ciccarelli FD, Doerks T, Von Mering C, Creevey CJ, Snel B, and Bork P. 2006. Toward automatic reconstruction of a highly resolved tree of life. Science 311:1283-1287.

Cole JR, Wang Q, Fish JA, Chai B, McGarrell DM, Sun Y, Brown CT, Porras-Alfaro A, Kuske CR, and Tiedje JM. 2013. Ribosomal Database Project: data and tools for high throughput rRNA analysis. Nucleic acids research 42:D633-D642.

De Queiroz K, and Gauthier J. 1994. Toward a phylogenetic system of biological nomenclature. Trends in Ecology \& Evolution 9:27-31. 10.1016/0169-5347(94)90231-3

DeSantis TZ, Hugenholtz P, Larsen N, Rojas M, Brodie EL, Keller K, Huber T, Dalevi D, Hu P, and Andersen GL. 2006. Greengenes, a chimera-checked $16 \mathrm{~S}$ rRNA gene database and workbench compatible with ARB. Applied and environmental microbiology 72:5069-5072.

Driskell AC, Ané C, Burleigh JG, McMahon MM, O'Meara BC, and Sanderson MJ. 2004. Prospects for building the tree of life from large sequence databases. Science 306:1172-1174.

Felsenstein J. 1985. Phylogenies and the comparative method. American Naturalist 125:1-15.

Fundel K, and Zimmer R. 2006. Gene and protein nomenclature in public databases. Bmc Bioinformatics 7:1.

Gouy M, and Delmotte S. 2008. Remote access to ACNUC nucleotide and protein sequence databases at PBIL. Biochimie 90:555-562. 10.1016/j.biochi.2007.07.003

Gouy M, Gautier C, Attimonelli M, Lanave C, and Di Paola G. 1985. ACNUC-a portable retrieval system for nucleic acid sequence databases: logical and physical designs and usage. Computer applications in the biosciences: CABIOS 1:167-172.

Hansen TF. 1997. Stabilizing selection and the comparative analysis of adaptation. Evolution 51:13411351.

Hebert PD, Cywinska A, and Ball SL. 2003. Biological identifications through DNA barcodes. Proceedings of the Royal Society of London B: Biological Sciences 270:313-321.

Hey J. 1992. Using phylogenetic trees to study speciation and extinction. Evolution:627-640.

Hollingsworth PM, Forrest LL, Spouge JL, Hajibabaei M, Ratnasingham S, van der Bank M, Chase MW, Cowan RS, Erickson DL, Fazekas AJ, Graham SW, James KE, Kim K-J, Kress WJ, Schneider H, van AlphenStahl J, Barrett SCH, van den Berg C, Bogarin D, Burgess KS, Cameron KM, Carine M, Chacón J, Clark A, Clarkson JJ, Conrad F, Devey DS, Ford CS, Hedderson TAJ, Hollingsworth ML, Husband BC, Kelly L, Kesanakurti PR, Kim JS, Kim Y-D, Lahaye R, Lee H-L, Long DG, Madriñán S, 
Maurin O, Meusnier I, Newmaster SG, Park C-W, Percy DM, Petersen G, Richardson JE, Salazar GA, Savolainen V, Seberg O, Wilkinson MJ, Yi D-K, and Little DP. 2009. A DNA barcode for land plants. Proceedings of the National Academy of Sciences 106:12794-12797. 10.1073/pnas.0905845106

Hollingsworth PR, Simons AM, Fordyce JA, and Hulsey CD. 2013. Explosive diversification following a benthic to pelagic shift in freshwater fishes. BMC Evol Biol 13. doi:10.1186/1471-2148-13-272

Izquierdo-Carrasco F, Cazes J, Smith SA, and Stamatakis A. 2014. PUmPER: phylogenies updated perpetually. Bioinformatics 30:1476-1477. 10.1093/bioinformatics/btu053

Katoh K, and Standley DM. 2013. MAFFT Multiple Sequence Alignment Software Version 7: Improvements in Performance and Usability. Mol Biol Evol 30:772-780. 10.1093/molbev/mst010

Kress WJ, Wurdack KJ, Zimmer EA, Weigt LA, and Janzen DH. 2005. Use of DNA barcodes to identify flowering plants. Proc Natl Acad Sci U S A 102:8369-8374. 10.1073/pnas.0503123102

Lassmann T, and Sonnhammer EL. 2005. Automatic assessment of alignment quality. Nucleic acids research 33:7120-7128.

Leslie AB, Beaulieu JM, Rai HS, Crane PR, Donoghue MJ, and Mathews S. 2012. Hemisphere-scale differences in conifer evolutionary dynamics. Proceedings of the National Academy of Sciences 109:16217-16221.

Maddison WP. 2006. Confounding asymmetries in evolutionary diversification and character change. Evolution 60:1743-1746.

Martin SD, and Bonett RM. 2015. Biogeography and divergent patterns of body size disparification in North American minnows. Mol Phylogenet Evol 93:17-28. 10.1016/j.ympev.2015.07.006

Mitchell J, McCray A, and Bodenreider O. 2003. From phenotype to genotype: issues in navigating the available information resources. Methods of information in medicine 42:557-563.

Morgan AA, Hirschman L, Colosimo M, Yeh AS, and Colombe JB. 2004. Gene name identification and normalization using a model organism database. Journal of biomedical informatics 37:396-410.

NCBI Resource Coordinators. 2017. Database Resources of the National Center for Biotechnology Information. Nucleic acids research 45:D12.

Paradis E, Claude J, and Strimmer K. 2004. APE: Analyses of Phylogenetics and Evolution in R language. Bioinformatics 20:289-290.

Perl Development Team. 2017. 5.26.

Python Software Foundation. 2016. Python Language. Version 3.5.4.

Quinn T. 2017. peakRAM: Monitor the Total and Peak RAM Used by an Expression or Function. R package version 1.0.2.

R Development Core Team. 2018. R: A language and environment for statistical computing. Vienna, Austria: R Foundation for Statistical Computing. Available at http://www.R-project.org/.

Rabosky DL, Santini F, Eastman J, Smith SA, Sidlauskas B, Chang J, and Alfaro ME. 2013. Rates of speciation and morphological evolution are correlated across the largest vertebrate radiation. Nat Commun 4:1958. 10.1038/ncomms2958

Ratnasingham S, and Hebert PD. 2013. A DNA-based registry for all animal species: the barcode index number (BIN) system. PLoS One 8:e66213. 10.1371/journal.pone.0066213

Ratnasingham S, and Hebert PDN. 2007. BOLD: The Barcode of Life Data System (www.barcodinglife.org). Molecular Ecology Notes 7:355-364. 10.1111/j.14718286.2006.01678.x

Schoch CL, Seifert KA, Huhndorf S, Robert V, Spouge JL, Levesque CA, Chen W, Bolchacova E, Voigt K, and Crous PW. 2012. Nuclear ribosomal internal transcribed spacer (ITS) region as a universal DNA barcode marker for Fungi. Proceedings of the National Academy of Sciences 109:6241-6246.

Schoenhuth S, Shiozawa DK, Dowling TE, and Mayden RL. 2012. Molecular systematics of western North American cyprinids (Cypriniformes: Cyprinidae). Zootaxa:281-303. 
Schofl G. 2015. reutils: Talk to the NCBI EUtils. R package version 0.2.2.

Shi JJ, and Rabosky DL. 2015. Speciation dynamics during the global radiation of extant bats. Evolution 69:1528-1545.

Smith SA, Beaulieu JM, and Donoghue MJ. 2009. Mega-phylogeny approach for comparative biology: an alternative to supertree and supermatrix approaches. BMC Evol Biol 9:37. 10.1186/1471-2148-937

Spriggs EL, Christin P-A, and Edwards EJ. 2014. C 4 photosynthesis promoted species diversification during the Miocene grassland expansion. PLoS One 9:e97722.

Stajich JE, Block D, Boulez K, Brenner SE, Chervitz SA, Dagdigian C, Fuellen G, Gilbert JG, Korf I, and Lapp H. 2002. The Bioperl toolkit: Perl modules for the life sciences. Genome Research 12:1611-1618.

Stamatakis A. 2014. RAxML version 8: a tool for phylogenetic analysis and post-analysis of large phylogenies. Bioinformatics 30:1312-1313. 10.1093/bioinformatics/btu033

Tamames J, and Valencia A. 2006. The success (or not) of HUGO nomenclature. Genome Biology 7:1.

Tautz D, Arctander P, Minelli A, Thomas RH, and Vogler AP. 2003. A plea for DNA taxonomy. Trends in Ecology \& Evolution 18:70-74.

Tuason O, Chen L, Liu H, Blake JA, and Friedman C. 2003. Biological nomenclatures: a source of lexical knowledge and ambiguity. Proceedings of the Pacific Symposium of Biocomputing. $p 238$.

Webb CO, Ackerly DD, McPeek MA, and Donoghue MJ. 2002. Phylogenies and community ecology. Annual Review of Ecology and Systematics:475-505.

Winter D. 2016. rentrez: Entrez in R. R. 0.2.4.

Zanne AE, Tank DC, Cornwell WK, Eastman JM, Smith SA, FitzJohn RG, McGlinn DJ, O'Meara BC, Moles AT, Reich PB, Royer DL, Soltis DE, Stevens PF, Westoby M, Wright IJ, Aarssen L, Bertin RI, Calaminus A, Govaerts R, Hemmings F, Leishman MR, Oleksyn J, Soltis PS, Swenson NG, Warman $\mathrm{L}$, and Beaulieu JM. 2014. Three keys to the radiation of angiosperms into freezing environments. Nature 506:89-92. 10.1038/nature12872 